\title{
Relativistic jet motion in the core of the radio-loud quasar $\mathrm{J} 1101+7225$
}

\author{
J.-U. Pott ${ }^{1,2}$, A. Eckart ${ }^{1}$, M. Krips ${ }^{1,3}$, T.P. Krichbaum ${ }^{4}$, S. Britzen ${ }^{4}$, W. Alef ${ }^{4}$, and J.A. Zensus ${ }^{4}$ \\ 1 I. Physikalisches Institut, University of Cologne, Zülpicher Strasse 77, 50939 Köln, Germany \\ e-mail: [pott; eckart;krips]@ph1.uni-koeln.de \\ 2 European Southern Observatory, Karl-Schwarzschild-Str. 2, 85748 Garching b. München, Germany \\ 3 Institut de Radio-Astronomie Millimétrique (IRAM), 300 rue de la piscine, 38406 Saint-Martin-d'Hères, France \\ 4 Max-Planck-Institut für Radioastronomie, Auf dem Hügel 69, 53121 Bonn, Germany \\ e-mail: [tkrichbaum; sbritzen;walef; azensus] @mpifr-bonn.mpg.de
}

Received 22 November 2004 / Accepted 25 February 2005

\begin{abstract}
Multi-epoch GHz Very Long Baseline Interferometry (VLBI) data of the radio-loud quasar J1101+7225 were analyzed to estimate the proper motion of extended optically thin jet components. Two components separated from the core could be mapped at $1.66 \mathrm{GHz}$, which is consistent with earlier observations. In one case we found evidence of high apparent superluminal motion $\left(\beta_{\text {app }}=22.5 \pm 4\right)$ at large (deprojected) distances to the core $(22 \mathrm{mas} \sim 4 \mathrm{kpc}$, at $z=1.46)$. Typically in other quasars such high separation velocities are only found much closer to the core component. Furthermore the Doppler factor, the magnetic field strength, and the angular size of the optically thick core were derived using published X-ray data. Analysis of $5 \mathrm{GHz}$ VLBI data reveals the existence of further jet components within the central 5 mas. Additionally the data published so far on the GHz-spectrum were discussed at all angular resolutions. J1101+7225 turns out to be a standard quasar for studying different aspects of radio jet kinematics out to kpc-scales.
\end{abstract}

Key words. galaxies: active - galaxies: jets - galaxies: kinematics and dynamics - quasars: individual: J1101+7225 galaxies: nuclei

\section{Introduction}

The radio-loud quasar $\mathrm{J} 1101+7225$ is a source of the Very Long Baseline Array (VLBA) Calibrator Survey VCS1 for phasereferencing observations (Beasley et al. 2002, in the following B02). We observed this source within larger experiments, that were focused on imaging faint Seyfert galaxies. It turned out that J1101+7225 shows a complex source structure, which makes it physically interesting but, on the other hand, also less suitable as a calibrator. Since so far very little information is available for individual sources of the VCS1, we present our results in this article. Analysis of the (relativistic) kinematics of non-thermal radio sources gives important physical insight into the inmost regions of an active galactic nucleus (AGN). The ejection of radio jet components and the jet kinematics are most probably related to the process of accretion onto the nucleus itself. Quantitative estimates of physical properties, such as magnetic fields and source sizes, can be derived, too. Our analysis is based on comparison of our $1.66 \mathrm{GHz}$ Very Long Baseline Interferometry (VLBI) map obtained in 2002 with the available maps of past observations (Thakkar et al. 1995 (T95) at $1.66 \mathrm{GHz}$ and B02 at $2.3 \mathrm{GHz}$ ). Further data from a multiepoch study at $5 \mathrm{GHz}$ of the central 5 mas of J1101+7225 by Britzen (2002) and Britzen et al. (in prep.) were used to
Table 1. General properties of $\mathrm{J} 1101+7225$.

\begin{tabular}{llc}
\hline \hline Property & Value & Ref. \\
\hline IAU $^{a} /$ other name & $\mathrm{J} 1101+7225 /[\mathrm{HB} 89] 1058+726$ & \\
RA $(\mathrm{J} 2000)$ & $11 \mathrm{~h} 01 \mathrm{~m}(48.8054 \pm 0.0001) \mathrm{s}$ & $\mathrm{B} 02$ \\
Dec $(\mathrm{J} 2000)$ & $+72^{\circ} 25^{\prime}\left(37.1183 \pm 0.6 \times 10^{-3}\right)^{\prime \prime}$ & $\mathrm{B} 02$ \\
Redshift & 1.46 & (b) \\
abs. magnitude & $-27.3 \mathrm{mag} \approx 10^{46.4} \mathrm{erg} \mathrm{s}^{-1}$ & (c) \\
$1.4 \mathrm{GHz}$ radio cont & $(1451 \pm 30) \mathrm{mJy}$ & (d) \\
\hline
\end{tabular}

a International Astronomical Union; (b): Jackson \& Browne (1991); (c): Véron-Cetty \& Véron (2001); (d): White \& Becker (1992).

determine the source structure of the central region, unresolved at lower observing frequencies.

In Table 1 the general properties of $\mathrm{J} 1101+7225$ are summarized to introduce the quasar. B02 estimated the position of J1101+7225 with (sub)mas-accuracy (Table 1), which is necessary for high-sensitivity phase-referencing experiments. In Sect. 2 details are given concerning the VLBI observations that are the basis for this article. Flux densities, the resulting maps, and a kinematical analysis of the mapped structure are 
Table 2. Details of the observing schedule of the EVN observation with eight antennae of $\mathrm{J} 1101+7225$.

\begin{tabular}{ccc}
\hline \hline Date of observation & Obs. frequency & Bit rate \& type of samp. \\
13 Feb. 2002 & $1630.49 \mathrm{MHz}$ & 256 Mbps \& 2 bit \\
\hline No. \& bandwidth of IF & polarization & system and correlator: \\
4 DSB-IFs \& 16 MHz & LCP & MKIV at MPIfR, Bonn \\
\hline
\end{tabular}

presented in Sect. 3. Finally the results are discussed and summarized in Sect. 4.

\section{Observations}

The quasar J1101+7225 was observed as a calibrator source within a larger European VLBI Network (EVN) experiment. The observations were conducted during the period 13-14 Feb. 2002, 21h-09h UT, while the scans of J1101+7225 typically lasted for three minutes at a total observing time of $1.6 \mathrm{~h}$. We observed at $18 \mathrm{~cm}$ with the $100 \mathrm{~m}$ antenna of the Max-Planck Institut für Radioastronomie (MPIfR) at Effelsberg, Germany, the $76 \mathrm{~m}$ antenna at Jodrell Bank, UK, the $32 \mathrm{~m}$ antenna at Medicina, Italy, the $25 \mathrm{~m}$ antenna at Onsala, Sweden, the $25 \mathrm{~m}$ antennae at Shanghai and Urumqi, China, the $32 \mathrm{~m}$ antenna at Torun, Poland, and the $14 \times 25 \mathrm{~m}$ antenna array at Westerbork, the Netherlands. Details concerning the observing mode are given in Table 2. After the observations, the data were correlated at the VLBI correlator of the MPIfR in Bonn, Germany, and imported into the Astronomical Image Processing System (AIPS) via MK4IN (Alef \& Graham 2002). The data were fringe-fitted and calibrated in a standard manner with the AIPS package and imaged using the DIFMAP VLBI package (Pearson et al. 1994).

Further we present $5 \mathrm{GHz}$ VLBI maps of $\mathrm{J} 1101+7225$, obtained in VLBA and global VLBI observations as part of a multi-epoch VLBI study (the Caltech-Jodrell Bank flatspectrum sample CJF; Taylor et al. 1996; Britzen 2002). These observations aim at a statistical investigation of the kinematics of a complete sample of AGN (Britzen 2002; Britzen et al. in prep.). The sources were observed in 5.5 min snapshot observations to determine the position and motion of jet components in the central 5 mas. The data were recorded over $32 \mathrm{MHz}$ total bandwidth broken up into 4 baseband channels with 1-bit sampling. The recorded data were correlated in Socorro, USA.

In Sect. 3.2 we include the VLBI-maps of J1101+7225 published earlier to analyze the evolution of the structure found. T95 observed the quasar with global VLBI at $1.66 \mathrm{GHz}$ in the CJ1 survey which later on became a part of the CJF sample. The other map at $2.3 \mathrm{GHz}$ was reduced from VLBA data (B02) via automatic imaging using the Caltech DIFMAP package.

\section{Radio properties of the quasar $\mathrm{J} 1101+7225$}

\subsection{Flux densities}

The flux density of J1101+7225 has been measured occasionally during the last two decades. In Table 3 we summarize the
Table 3. Measured radio flux densities or peak brightnesses of $\mathrm{J} 1101+7225$, obtained with single-dish telescopes, local interferometers, and VLBI. The VLBI flux densities are our fitted core flux densities as presented in Tables 5 and 7.

\begin{tabular}{cccc}
\hline \hline Obs. type & Freq. [GHz] & Flux dens. [mJy] & \\
\hline single-dish & 0.038 & $\left(1.41 \times 10^{4} \pm 800\right)$ & $(\mathrm{a})$ \\
single-dish & 0.178 & $(3900 \pm 600)$ & $(\mathrm{b})$ \\
single-dish & 1.4 & $(1451 \pm 30)$ & $(\mathrm{c})$ \\
single-dish & 2.7 & $(1070 \pm 35)$ & $(\mathrm{d})$ \\
single-dish & 5 & $(858 \pm 76)$ & $(\mathrm{e})$ \\
single-dish & 22 & $(820 \pm 100)$ & $(\mathrm{f})$ \\
\hline local interf. & 1.4 & $(748 \pm 40) / \mathrm{beam}$ & $(\mathrm{g})$, res $\sim 1.5^{\prime \prime}$ \\
local interf. & 8.4 & $(349 \pm 20) / \mathrm{beam}$ & $(\mathrm{h})$, res $\sim 0.2^{\prime \prime}$ \\
\hline VLBI & 1.66 & $(396 \pm 20)$ & EVN Feb. 2002 \\
VLBI & 2.3 & $(520 \pm 25) / \mathrm{beam}$ & $\mathrm{B} 02$, res $\sim 3$ mas \\
VLBI & 5 & $(139 \pm 7)$ & $-1991.4-$ \\
VLBI & 5 & $(282 \pm 14)$ & $-1993.4-$ \\
VLBI & 5 & $(337 \pm 17)$ & $-1996.6-$ \\
VLBI & 5 & $(439 \pm 22)$ & $-1999.9-$ \\
VLBI & 8.4 & $(383 \pm 20) /$ beam & B02, res $\sim 1$ mas \\
\hline
\end{tabular}

References and comments: (a) Rees (1990), catalogue revised by Hales et al. (1995); (b) Gower et al. (1967); (c) White \& Becker (1992); (d) Kühr et al. (1981); (e) Gregory et al. (1996); (f) Teräsranta et al. (2001); (g) Xu et al.(1995) gave this peak brightness of a map, which was convolved to a circular beam of $1.5^{\prime \prime}$ FWHM; (h) Patnaik et al. (1992) found this peak brightness to be $80 \%$ of the total flux density of $436 \mathrm{mJy}$.

The values are plotted over frequency in Fig. 1. For the interferometric observations $5 \%$ flux density errors are given.

measurements at the various frequencies. Based on these data we calculated the spectrum, shown in Fig. 1. The single-dish observations show a curved radio spectrum with a steep spectrum at low $\mathrm{MHz}$ frequencies $\left(\alpha_{\text {single }}^{38 \mathrm{M} ; 178 \mathrm{M}} \sim-0.8 ; S \sim v^{\alpha}\right)$ and considerable flattening towards higher frequencies $\left(\alpha_{\text {single }}^{1.4 \mathrm{G} ; 5 \mathrm{G}} \sim\right.$ $\left.-0.4 ; \alpha_{\text {single }}^{5 \mathrm{G} ; 2 \mathrm{G}} \sim 0\right)$. The spectral trend of the Very Large Array (VLA) measurements follows the single dish measurements. The slightly flatter shape $\left(\alpha_{\text {locIF }}^{1.4 \mathrm{G} ; 8.4 \mathrm{G}} \sim-0.3\right)$ plotted in Fig. 1 may overestimate the real spectrum, because only the peak brightness is published at $1.4 \mathrm{GHz}$.

In contrast with the EVN we measured a core flux density of $(396 \pm 20) \mathrm{mJy}$ at $1.66 \mathrm{GHz}$. In combination with the core flux densities obtained at $5 \mathrm{GHz}$ and the lower flux density limits given by the peak brightnesses of B02, this confirms a flat spectral shape (within the scatter due to different observations and epochs) at GHz-frequencies, which is shown as a dotted line in Fig. 1. These findings indicate that the continuous flattening of the single-dish spectrum is evoked by superposition of spectrally steep components, still unresolved with local interferometry but fully resolved and/or separable at VLBI resolution, and by the flat VLBI core, which is still nearly unresolved at the observing frequencies of $1.66 \mathrm{GHz}$ 


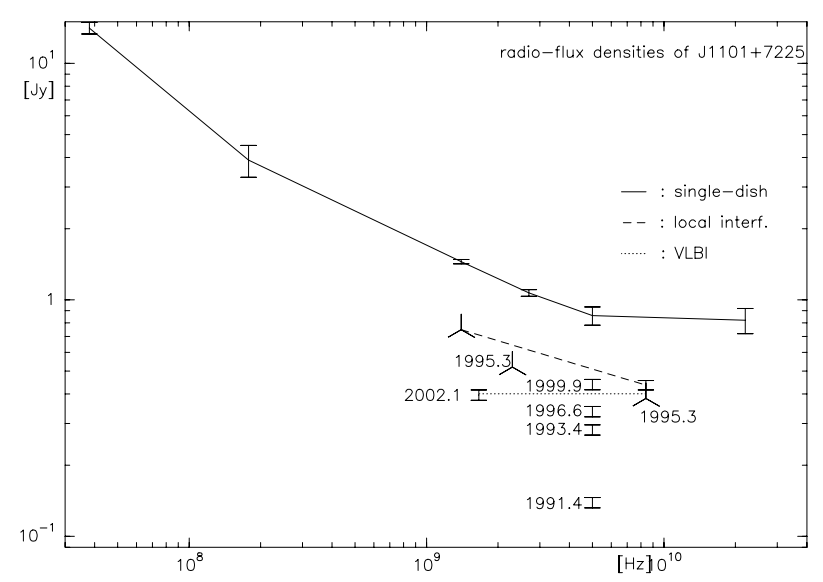

Fig. 1. The radio spectrum of J1101+7225 at different angular resolutions, corresponding to Table 3. All VLBI measurements are labelled with the observing date. While the flux densities are plotted with error bars, the stars indicate peak brightnesses. Because the peak brightnesses may underestimate the flux densities, the corresponding spectra are dotted / dashed (see Sect. 3.1).

Table 4. The published single-dish flux densities at $5 \mathrm{GHz}$ to investigate the source variability. (1) observed at $4.9 \mathrm{GHz}$ and corrected to $5 \mathrm{GHz}$ via a spectral index $\alpha_{2.7 \mathrm{GHz}}^{10.7 \mathrm{GHz}}$ (2) at $4.85 \mathrm{GHz}$; (3) at $4.75 \mathrm{GHz}$; (a): Kühr et al. (1981); (b): Gregory et al. (1996); (c): Gregory \& Condon (1991); (d): Reich et al. (2000).

\begin{tabular}{cccc}
\hline \hline$S_{5 \mathrm{GHz}}[\mathrm{mJy}]$ & Date of observation & Ref. & Ann. \\
\hline $778 \pm 4$ & between Feb. 1977 and Mar. 1978 & (a) & (1) \\
$788 \pm 31$ & Nov. 1986 & (b) & (2) \\
$953 \pm 73$ & Oct. 1987 & (c) & (2) \\
$623 \pm 56$ & $1992.5=$ Jul. 1992 & (d) & (3) \\
\hline
\end{tabular}

and $2.3 \mathrm{GHz}$. Königl (1981) calculated such flat GHz-spectra of compact synchrotron sources, involving optical thickness for the synchrotron radiation due to synchrotron self-absorption. Thus most of the central radio emission of $\mathrm{J} 1101+7225$ at these frequencies is emitted by a compact, unresolved core.

A significant fraction (up to $50 \%$ ) of the single-dish flux density of the whole galaxy is radiated by the inmost region, still unresolved by interferometric observations. Relativistic beaming models explain the extraordinary luminosity of radioloud cores with unresolved radio-jet components approaching nearly along the line of sight at relativistic velocities (e.g. Blandford \& Königl 1979, and see Sect. 3.4).

One consequence of these models is a straightforward explanation of the often observed variability of the core flux density by small variations in the jet orientation with respect to the observer. This variability is still observable with singledish observations in case of core-dominated $\mathrm{GHz}$ emission. Table 4 presents single-dish radio flux densities, measured at $5 \mathrm{GHz}$ at different epochs. The values show a flux density variation up to $20 \%$ between 1986 and 1987 . This variability has to be taken into account and leads to significant changes of

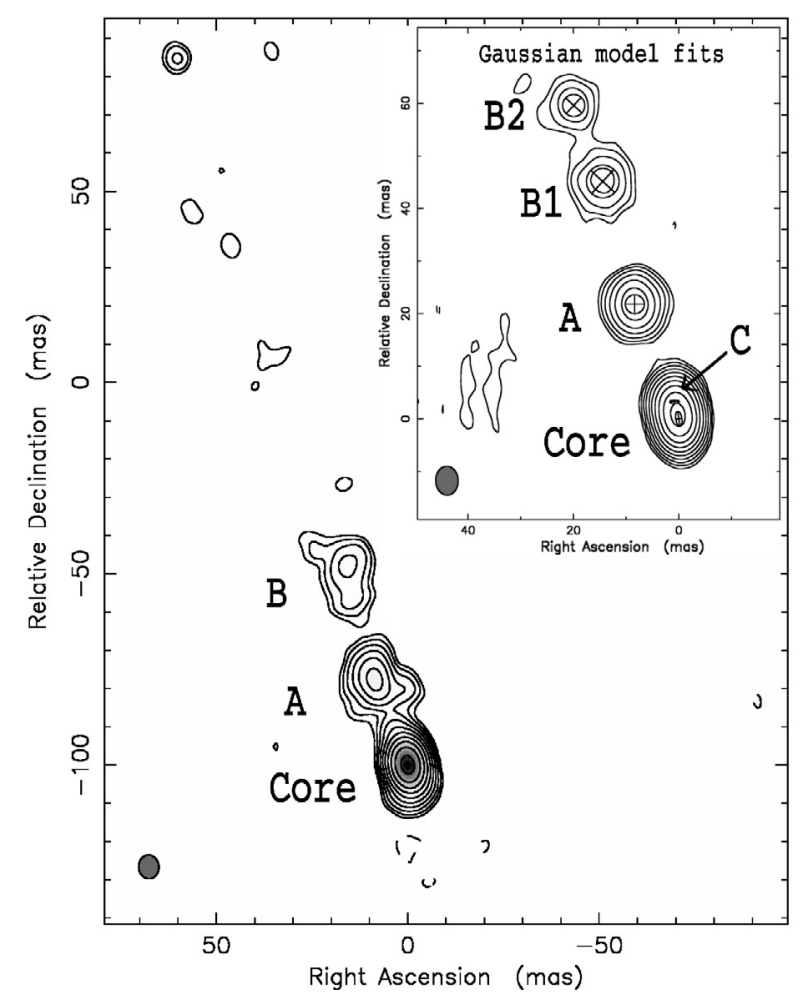

Fig. 2. Cleaned map of the EVN observation of $\mathrm{J} 1101+7225$ at $1.66 \mathrm{GHz}$. The map shows a peak brightness of $407 \mathrm{mJy} / \mathrm{beam}$. The contour levels are $-0.15,0.15,0.3,0.6,1.2,2.4,4.8,9.6,19.2,38.4$, $76.8 \%$ of the peak brightness, and the beam size is $6.22 \times 5.28$ mas at position angle (PA) of $4^{\circ}$. In the upper right corner the Gaussian model fit representation of the VLBI image is given (with the same contour levels, but now with respect to a $399 \mathrm{mJy} /$ beam peak brightness), obtained by fitting Gaussian components to the visibility data (beam: $5.5 \times 4.3$ mas at PA $2^{\circ}$ ). By this technique the extended structure at $\sim 50$ mas distance to the core could be clearly resolved into two components.

short-range ${ }^{1}$ spectral indices in the $\mathrm{GHz}$ domain from different observing epochs. The underlying variability of the VLBI core flux density was observed at $5 \mathrm{GHz}$ (Table 3 ) and shows the same minimum at around 1992. Therefore only the later measurements were taken in Fig. 1 to approximate the spectral index of the VLBI core. The observed flux density variability of the core component strongly supports the concept of beamed radio emission, which can be confirmed in Sect. 3.4.

\subsection{High apparent superluminal motion outside the central 10 mas}

In Fig. 2 we present the map made from the EVN observation in Feb. 2002. Clearly two extended regions are visible beside the core with more than $4.8 \%$ and $1.2 \%$ of the core peak brightness. They are labeled with A and B, respectively. More information about the underlying source structure can be retrieved by fitting circular and elliptical Gaussians to the visibility data

\footnotetext{
1 Due to the power-law relation between flux density and frequency, the influence of the flux density variability on the spectral index decreases with increasing frequency interval.
} 
Table 5. The best fit Gaussians as mentioned in the text. $a$ labels the major axis of elliptical Gaussians. A, B1, and B2 were only fitted by circular Gaussians, because the locations of components of lower flux density can be fitted more reliably with less degrees of freedom. The flux density errors are $\sim 5 \%$, the uncertainties of the location, and size of the Gaussians range between $0.2-0.5$ of the beamsize depending on the respective flux density.

\begin{tabular}{cccccc}
\hline \hline & $\begin{array}{c}\text { Flux dens. } \\
(\mathrm{mJy})\end{array}$ & \multicolumn{2}{c}{ Location } & \multicolumn{2}{c}{ Size } \\
& $r(\mathrm{mas})$ & $\vartheta(\mathrm{deg})$ & $a(\mathrm{mas})$ & Axial ratio \\
\hline Core & 396 & 0 & 0 & 2.88 & $0.41 @ 9.4^{\circ}$ \\
$\mathrm{C}$ & 149 & 3.3 & 14.1 & 1.83 & $0.39 @-89.4^{\circ}$ \\
$\mathrm{A}$ & 54 & 23.3 & 21.0 & 3.62 & 1 \\
$\mathrm{~B} 1$ & 16 & 47.4 & 17.7 & 6.22 & 1 \\
$\mathrm{~B} 2$ & 7 & 62.8 & 18.5 & 4.21 & 1 \\
\hline
\end{tabular}

and optimizing the fit to the amplitudes and closure phases (cf. Pearson 1995). The structure in 200 mas distance to the core in N-NE direction could be confirmed neither by applying different taper to the uv data nor by a respective model component. Our best model of the source structure is given in Table 5 and the corresponding map is inserted in Fig. 2. The measurement errors on the model parameters flux density, relative position, and source size are given in the respective tables. Their estimation is based on the comparison of different model fits, as described by Gómez \& Marscher (2000). A more theoretical approach (Fomalont 1989) based on the dynamic range of the map gives comparable magnitudes.

This analysis shows that the extended B component from the cleaned map is a blend of at least two components (B1, B2). Furthermore within the central 5 mas a significant fraction of the flux density is radiated by an extended source beside the dominating unresolved core at a distance of a few mas to the core (see Sect. 3.3). This region close to the limit of resolution of the $1.66 \mathrm{GHz}$ observations is studied in more detail in the next Sect. (3.3) at higher frequencies.

T95 detected both A and B, too. A detailed comparison of our data with the Gaussian component model of T95 reveals a high separation velocity of the A-component at an unusually large deprojected distance to the core. In contrast to Table 5, they could fit three elliptical Gaussian components to the region around $\mathrm{A}$ and one to the $\mathrm{B}$ region. Because the angular extensions of both regions (A and $\mathrm{B}$ ) are close to the limit of resolution, we prefer the blend interpretation of this situation. Our A component does not correspond to one of the three fitted components of T95 but does correspond to a flux densityweighted mean of all three components; and vice versa, the mean of our B1 and B2 components corresponds to the one component of that region fitted by T95 to their visibility data. Although the full width at half maximum (FWHM) of the circular Gaussians B1 and B2 (cf. Table 5) are not overlapping, the modeling process showed that due to their low flux densities, the sizes of the $\mathrm{B}$ components are insecure up to a factor of two. It is also possible that a third component exists, slightly too weak to be fitted. Thus the used flux density-weighted mean appears to be the most adequate presentation.
We determined, therefore, the apparent motion of the radio jet structures $\mathrm{A}$ and $\mathrm{B}$ with respect to the core (Table 6), where in case of several fit components the labels refer to the flux density-weighted mean of these. Angular velocities $\mu$ are presented in Table 6 on the right side. They are confirmed by the snapshot VLBA map at $2.3 \mathrm{GHz}$ of B02 (last column in Table 6). We did not directly include these data in the velocity calculation. The introduced uncertainties due to the different observing frequency and the lack of a Gaussian modelfit would annihilate the increased calculation accuracy. To demonstrate the apparent angular motion, we show the three VLBI maps in one figure in Fig. 3 on a common angular scale and rotated by about $-20^{\circ}$.

The dimensionless linear equivalents $\beta_{\text {app }}$ are calculated via $\beta_{\text {app }}=\mu \frac{z}{H_{0}(1+z)}\left[\frac{1+\sqrt{1+2 q_{0} z}+z}{1+\sqrt{1+2 q_{0} z}+q_{0} z}\right]$

(cf. Pearson \& Zensus 1987). The measured apparent superluminal transverse motion can be transformed via special relativity $^{2}$ into a minimal intrinsic velocity, expressed as:

$\beta_{\min }^{\mathrm{A}} \approx 0.9990_{-0.0005}^{+0.0003}$ at $\vartheta_{\text {min }}^{\mathrm{A}} \approx 2.54_{-0.38}^{\circ+0.55}$.

The 22 mas distance to the core at $\vartheta=2.5^{\circ}$ can be deprojected to a large linear distance of about $4 \mathrm{kpc}$ (at $z=1.46, q_{0}=0.1$ ). It turns out that the uncertainties in the estimation of $\beta_{\text {app }}$ and the Hubble parameter are affecting $\beta_{\text {min }}^{\mathrm{A}}$ by less than $0.1 \%$. The more distant B-component was found to be stationary within the errors at a mean angular distance of 52.9 mas.

\subsection{Resolved structure in the central 10 mas}

The higher-resolution maps of the $5 \mathrm{GHz}$ snapshots of Britzen et al. (in prep.) are presented in Fig. 4. They explicitly show that the Core region of the $1.66 \mathrm{GHz}$ map is not totally compact on the 1 mas scale of the $5 \mathrm{GHz}$ observations. Extended Gaussian components could be fitted to the data beside the dominant ${ }^{3}$ central source. This was already suggested by the C-component, fitted to the $1.66 \mathrm{GHz}$ data (Table 5), and it confirms the power of the model fits in analysing the data.

In Table 7 the fitted Gaussian components are shown. A linear fit of the core-distance with respect to the observing epochs (Fig. 5) demonstrates the apparently superluminal motion of both components and shows mean separation velocities of

$\beta_{\mathrm{app}}^{\mathrm{C} 1} \approx(2.5 \pm 2.5) \quad$ and $\quad \beta_{\mathrm{app}}^{\mathrm{C} 2} \approx(3.7 \pm 2.5)$.

The given standard deviations of the fitted velocities are relatively large due to the few available data points. Because a significant intrinsic acceleration of the jet material is very unlikely from the 4 mas regime of the inner C-components toward the 20 mas regime far away from the central engine, we

\footnotetext{
${ }^{2}$ If $\vartheta$ is the angle enclosed by the line of sight and the direction of motion and if $\beta$ is the intrinsic velocity, one finds $\beta_{\text {app }}=\frac{\beta \sin \vartheta}{1-\beta \cos \vartheta}$. The minimal intrinsic velocity $\beta_{\min }=\sqrt{\left(\beta_{\mathrm{app}}^{2}\right) /\left(1+\beta_{\mathrm{app}}^{2}\right)}$ implies an angle $\vartheta_{\text {min }}$ fulfilling: $\cot \left(\vartheta_{\text {min }}\right)=\beta_{\text {app }}$.

3 E.g. at the last epoch the extended components show flux densities of $20 \%$ of the central flux density.
} 
Table 6. The angular distances between the core and the fitted Gaussian jet components (or their flux density weighted mean; cf. text). The errors reflect uncertainties in the fitting process and increase with decreasing component flux density, while derivation of the apparent velocities is described in the text. The position angles of the components did not change significantly during the observing epochs and were omitted. In the last column the read out values from a map by B02 are given for comparison, but cannot be included in the calculations due to lack of a Gaussian model fit.

\begin{tabular}{c|cc|cc|c}
\hline \hline Epoch \& observ. freq. [GHz]: & 25 Sep. 1991 at 1.66 [T95] & 13 Feb. 2002 at 1.66 & \multicolumn{2}{c|}{ Mean App. Transv. velocity } & 19 Apr. 1995 at 2.3 \\
\hline components: & \multicolumn{1}{c|}{ Distances in Fig. 3 [mas] } & $\mu\left[\mathrm{mas} \mathrm{yr}^{-1}\right.$ ] & $\beta_{\text {app; } h=0.71}{ }^{b}$ & Dist. in Fig. 3 [mas] \\
Core- $A$ & $(19.6 \pm 0.5)$ & $(23.3 \pm 0.5)$ & $(0.36 \pm 0.07)$ & $(22.5 \pm 4)$ & $(21.5 \pm 3)$ \\
Core- $B$ & $(53.7 \pm 1)$ & $(52.1 \pm 1)$ & $0^{a}$ & 0 & $(52 \pm 5)$ \\
\hline
\end{tabular}

${ }^{a}$ No significant propagation of the $\mathrm{B}$ component was found with respect to the errors.

${ }^{b}$ For the calculations we used $H_{0}=71_{-3}^{+4} \mathrm{~km} \mathrm{~s}^{-1} \mathrm{Mpc}^{-1}$ and a deceleration parameter $q_{0}=0.1$.

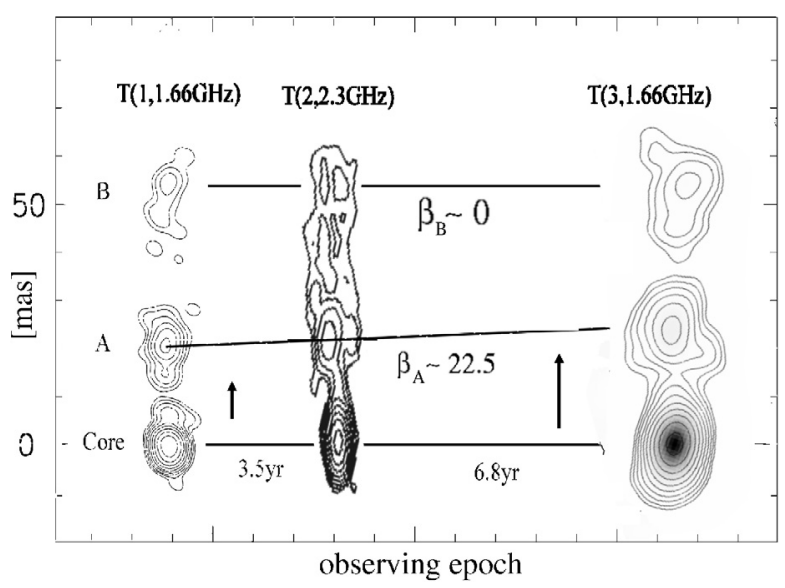

Fig. 3. The cleaned maps are aligned vertically (rotation about $-20^{\circ}$ ), which is justified by the fact, that no significant change of the position angle of the jet axis was observed. The apparent linear velocities of the fitted Gaussian components (cf. Table 6) are given. The first map by $\mathrm{T} 95$ at $\mathrm{T}(1,1.66 \mathrm{GHz})$ shows a peak brightness of $476 \mathrm{mJy} / \mathrm{beam}$, the contour levels are $-2,-1,1,2,4,8,16,32,63,127,253 \mathrm{mJy} / \mathrm{beam}$, and the beam size is $4.2 \times 3.2$ mas at PA $90^{\circ}$. The second map by B02 at $\mathrm{T}(2,2.3 \mathrm{GHz})$ shows a peak brightness of $520 \mathrm{mJy} / \mathrm{beam}$, the contour levels are $-3,3,6,12,24,48,96,192,384 \mathrm{mJy} / \mathrm{beam}$, and the beam size is $3.3 \times 6.7$ mas at PA $20^{\circ}$. The beam PAs given here refer to the unrotated coordinate system.

adopt the $\beta_{\mathrm{min}}^{\mathrm{A}} \approx 0.999$ from Eq. (1) as the intrinsic separation velocity also for the inner jet components. From the observed apparent separation velocities of $\mathrm{C} 1$ and $\mathrm{C} 2$ one uses the equations of special relativity ${ }^{2}$ to calculate two possible jet orientation angles $\vartheta_{s, l}$ to the line-of-sight for each component (with $\beta_{\min }^{\mathrm{A}}=0.999_{-0.0005}^{+0.0003}$ ):

$\beta_{\mathrm{app}}^{\mathrm{C} 1} \approx(2.5 \pm 2.5): \quad \vartheta_{s}^{\mathrm{C} 1} \in\left[0^{\circ}, 0.45^{\circ}\right], \quad \vartheta_{l}^{\mathrm{C} 1} \in\left[22^{\circ}, 180^{\circ}\right]$

$\beta_{\mathrm{app}}^{\mathrm{C} 2} \approx(3.7 \pm 2.5): \vartheta_{s}^{\mathrm{C} 2} \in\left[0.06^{\circ}, 0.56^{\circ}\right], \vartheta_{l}^{\mathrm{C} 2} \in\left[18^{\circ}, 80^{\circ}\right]$.

The uncertainty of $\beta_{\mathrm{app}}^{\mathrm{C} 1,2}$ dominates the errors of Eq. (3). For both components the calculated orientations differ significantly from $\vartheta_{\text {min }}^{\mathrm{A}} \approx\left(2.54_{-0.38}^{+0.55}\right)^{\circ}$ of Eq. (1).

A closer look at the modelfits in Table 7 reveals some trends over the observing epochs. The separation motion seems to decrease at later epochs in correlation with a decrease in the respective component brightnesses and an increasing position angle of $\mathrm{C} 1$ from $0.2^{\circ}$ to $7.8^{\circ}$ over the four epochs. With
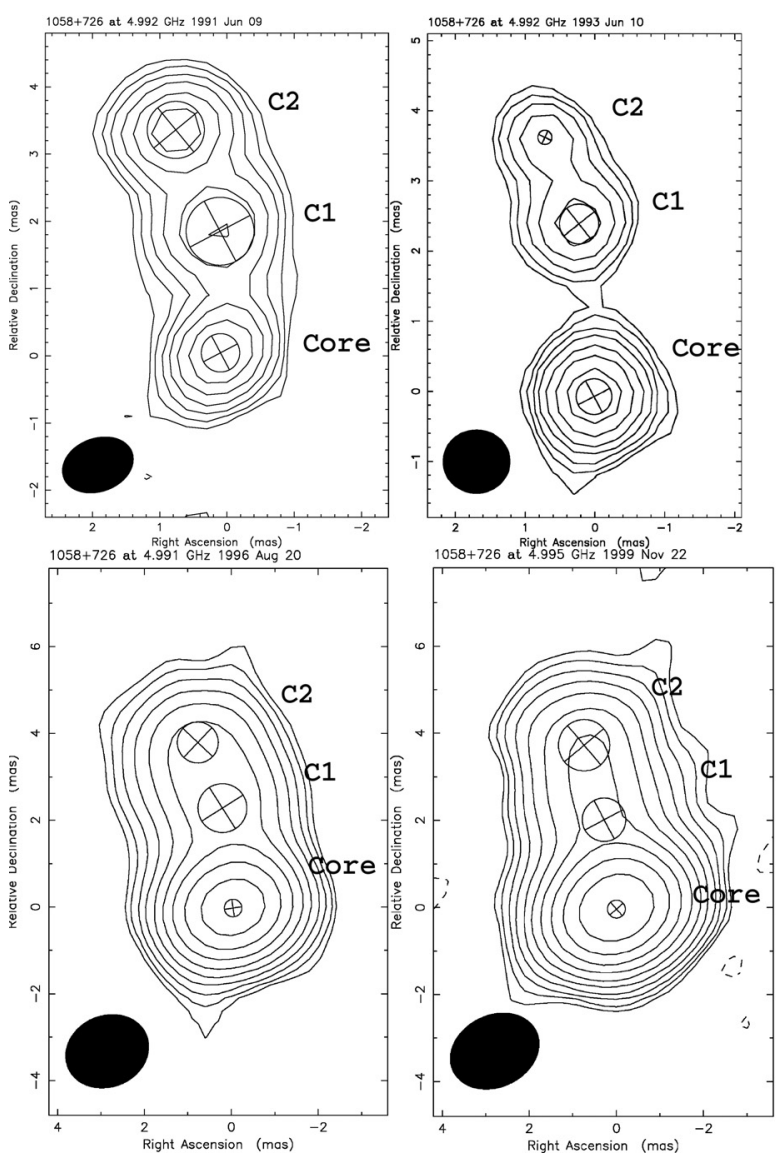

Fig. 4. $5 \mathrm{GHz}$ maps of the central 5 mas. The details are given in the form: peak brightness; contour levels in \%; beam size. First epoch: $0.097 \mathrm{mJy} / \mathrm{beam}$; levels are $-8,8,12,18,27,40.5,60.8 \%$; beam: $1.08 \times 0.79$ mas at PA $-70^{\circ}$. Second epoch: $0.209 \mathrm{mJy} /$ beam; levels are $6,9,13.5,20.3,30.4,45.6,68.3 \%$; beam: $0.96 \times 0.90$ mas at PA $-90^{\circ}$. Third epoch: $0.314 \mathrm{mJy} /$ beam; levels are 1.6, 2.56, 4.1, 6.55, 10.5, $16.8,26.8,42.9,68.7, \%$; beam: $1.95 \times 1.64$ mas at PA $-67^{\circ}$. Fourth epoch: $0.417 \mathrm{mJy} /$ beam; levels are $-0.9,0.9,1.44,2.3,3.69,5.9,9.44$, 15.1, 24.2, 38.7, 61.8\%; beam: $2.11 \times 1.64$ mas at PA $-65^{\circ}$. Crosses indicate the positions of circular Gaussian modelfit components. The detailed model parameters are presented in Table 7.

the given, relatively poor time-resolution of four observations within eight years it is not possible to fit these trends reliably. Nevertheless in combination with the different calculated angles in Eq. (1) and (3), they can be interpreted as indications 


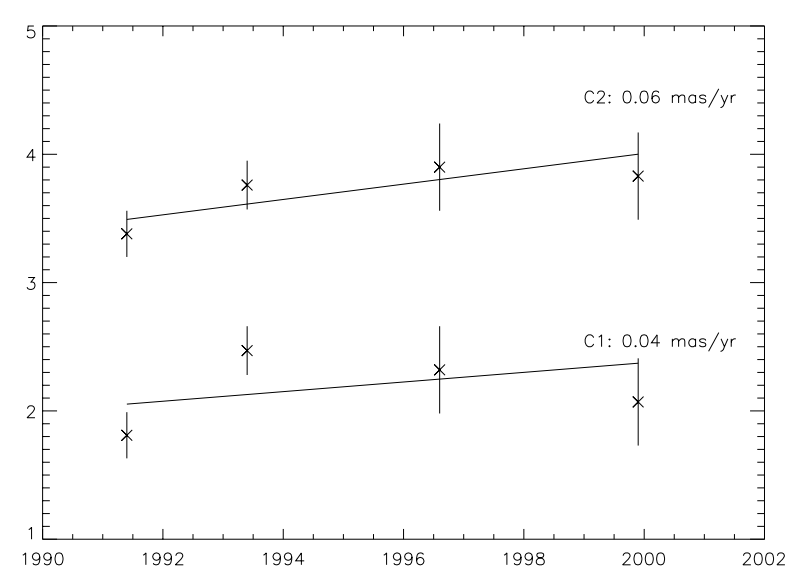

Fig. 5. Evolving core-distance (in mas) of the best fit Gaussians, taken from Table 7 over the years. A least-squares fit of a linear time-dependence is shown with respect to the errorbars. The standard deviation of the resulting apparent separation velocities is $\sigma \approx$ 0.04 mas $^{-1} r^{-1}$.

Table 7. The best fit circular Gaussians of the $5 \mathrm{GHz}$ data over four epochs. The flux density errors are $\sim 5 \%$, and the uncertainties of the location and size of the Gaussians range between $0.2-0.5$ of the beam size depending on the respective flux density.

\begin{tabular}{cccccc}
\hline \hline Epoch & & $\begin{array}{c}\text { Flux density } \\
(\mathrm{mJy})\end{array}$ & \multicolumn{2}{c}{ Location } & Size \\
& & (mas) & $\vartheta(\mathrm{deg})$ & diam (mas) \\
\hline 1991.4 & Core & 139 & 0 & 0 & 0.57 \\
& $\mathrm{C} 1$ & 132 & 1.81 & 0.2 & 1.01 \\
& $\mathrm{C} 2$ & 133 & 3.38 & 11.3 & 0.85 \\
\hline 1993.4 & Core & 282 & 0 & 0 & 0.51 \\
& $\mathrm{C} 1$ & 113 & 2.47 & 4.9 & 0.57 \\
& $\mathrm{C} 2$ & 61 & 3.76 & 10.7 & 0.20 \\
\hline 1996.6 & Core & 337 & 0 & 0 & 0.40 \\
& $\mathrm{C} 1$ & 97 & 2.32 & 6.0 & 1.13 \\
& $\mathrm{C} 2$ & 80 & 3.9 & 12.0 & 0.94 \\
\hline 1999.9 & Core & 439 & 0 & 0 & 0.41 \\
& $\mathrm{C} 1$ & 78 & 2.07 & 7.8 & 1.00 \\
& $\mathrm{C} 2$ & 84 & 3.83 & 11.1 & 1.18 \\
\hline
\end{tabular}

of a spatially curved jet structure. Different line-of-sight orientations account for different brightnesses, position angles, and apparent separation velocities.

The core flux density has strongly increased over the four epochs, which suggests that over the later epochs either a new jet component has emerged from the compact core region or the unresolved jet has changed its orientation with respect to the line of sight. During the fitting process we found indications that indeed a third component in sub-mas distance to the core is hidden; but probably due to its proximity to the core, this new component could not be fitted separately without doubts.

On the other hand, the increased brightness of the core at the later epochs may affect uncertainties in the model fitting process of the close $\mathrm{C}$ components. The values of one fifth of the beam size (cf. caption of Table 7 and discussion in Sect. 3.2), which are used for the linear fit in Fig. 5, may still slightly underestimate the real errors due to increased overblending of the $\mathrm{C}$ components by the close core radiation. Because this effect is not quantifiable with our data, we kept using these errors.

The idea of a jet curvature over the observed core-distances does not rule out a ballistic situation at the origin of the jet, as was described recently by Stirling et al. (2003). As origin of the radio jet in BL Lacertae they found a "precessing nozzle" which ejects the single components along straight trajectories. But outside 2 mas (corresponding to 0.4 mas at the redshift of $\mathrm{J} 1101+7225$ ) these trajectories became curved as well.

Summarizing the motion of all detected jet components including $\mathrm{A}$ and $\mathrm{B}$, the situation resembles the different measured apparent component speeds of the radio jet of the S5 quasar 0836+710 which extends over more than 150 mas at $z \sim 2.17$ (Hummel et al. 1992) and where no systematic correlation between the component speed and its distance to the core seems to be present (Otterbein et al. 1998, and references therein).

\subsection{Indirect estimation of relativistic bulk motion in the core}

Our VLBI-data of J1101+7225 allow, in combination with published data at other wavelengths, a straightforward analysis of the observed VLBI core flux density by applying a relativistic beaming model. The crucial idea of such models is that due to relativistic bulk motion of the sources, the radiation is amplified or attenuated significantly in the rest-frame of the observer, depending on the direction of motion with respect to the line of sight. Of course the bulk motion cannot be observed directly due to the lack of spatial resolution. But we share the common assumption that the X-ray emission of the core consists mainly of synchrotron photons scattered to shorter wavelengths via the inverse Compton effect. Marscher (1983) quantitatively derived the connection between radio and X-ray flux densities, their spectral indices, and the Doppler factor ${ }^{4} \delta$ for a homogeneous source of spherical shape. This implies that $\delta$ can be estimated from the other measured values.

In Table 8 the ingoing values of the calculations are given. The notation of the radio properties follows Marscher (1983). Typically the unresolved core consists of several components each of which dominates the unresolved spectrum at a different frequency (e.g. Marscher 1988). Therefore we can assume that the measured properties at $5 \mathrm{GHz}$ describe the turn-over point (index $m$ ) of one underlying source component and that the formulae can be applied for that component. The X-ray measurements were taken independently in the 1990s. Therefore a mean of the different $5 \mathrm{GHz}$ measurements is the most appropriate way to combine both datasets.

The mean measured Gaussian source size is transformed into $\theta^{\text {circ }}$ assuming an underlying circular source shape (see Marscher 1983 and Table 8). The angular source size should be

\footnotetext{
${ }^{4}$ With the terms of footnote 2 it is $\delta=(\gamma(1-\beta \cos \vartheta))^{-1}$ with $\gamma=1 / \sqrt{1-\beta^{2}}$.
} 
Table 8. The radio values characterize the compact core at $5 \mathrm{GHz}$ as discussed in Sect. 3.4. The turn-over properties are mean values of the model fits in Table 7. The cut-off frequency $v_{2}$ of the synchrotron spectrum is extrapolated from the optical-radio spectrum. The right column gives the calculated values.

\begin{tabular}{cc|c}
\hline \hline Radio & X-ray & Results \\
\hline$S_{\mathrm{m}} @ v_{\mathrm{m}} ; v_{2}$ & $\alpha_{\mathrm{X}}^{(1 \mathrm{keV} ; 5 \mathrm{keV}) a}$ & Doppler factor \\
$300 \pm 15 \mathrm{mJy} @ 5 \mathrm{GHz} ; 2 \mathrm{THz}$ & $-0.5 \pm 0.2$ & $\delta=0.5_{-0.3}^{+1.8}$ \\
\hline$\theta_{\mathrm{m}}^{\text {circ }}$ & $S_{\mathrm{obs}}^{\mathrm{X}}(1 \mathrm{keV})^{a}$ & Magnetic field $B_{0}$ \\
$(0.9 \pm 0.45) \mathrm{mas}$ & $0.1 \mu \mathrm{Jy}$ & $\left(0.15_{-0.12}^{+0.2}\right) \mathrm{G}$ \\
\hline
\end{tabular}

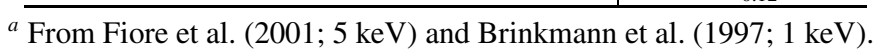

understood as an order of magnitude estimate ${ }^{5}$. Further we can use only the measured peak flux density $S_{\mathrm{m}}$ in contrast to the extrapolated one as foreseen by Marscher (1983). This results in a slight underestimation of $\delta$. The calculations lead to $\delta=$ $\left(0.5_{-0.3}^{+1.8}\right)$ and a magnetic field strength of $B_{0}=\left(0.15_{-0.12}^{+0.2}\right) \mathrm{G}$ of the unresolved synchrotron-self absorbed source (cf. Table 8).

However further radio components, peaking at higher frequencies, and other processes than inverse Compton scattering may contribute to the observed X-ray flux densities. This results in a probable underestimation of $\delta$ and $B_{0}$. In fact a flat to inverted spectral index as indicator for several components is suggested by the VCS1 map at $8.4 \mathrm{GHz}$ (B02). Although the lack of a detailed model fit inhibits reproducible calculations, visual inspection of the map reveals a dominating core, probably unresolved. Under the latter assumption the given peak brightness resembles the flux density of the component in mid 1995, which is slightly larger than the respective values of the $5 \mathrm{GHz}$ experiment at that time (cf. Fig. 1).

With the angular source size and the Doppler factor, the intrinsic brightness temperature can be calculated as

$T_{\text {intr }}=T_{\text {app }} / \delta=\left(2.8_{-0.2}^{+0.5} \cdot 10^{11} \mathrm{~K}\right)$.

These values fit well in the range of brightness temperature in which the simultaneously observed synchrotron emission and the inverse Compton-scattering are both effective enough to fulfill the inverse Compton scenario adopted here $\left(2 \times 10^{11} \mathrm{~K} \leq\right.$ $T_{\text {IC }} \leq 10^{12} \mathrm{~K}$; e.g. Kraus 1986; Bloom \& Marscher 1991). Without applying a Doppler factor the apparent brightness temperature at $5 \mathrm{GHz}$ rises from $0.5-3.1 \times 10^{11} \mathrm{~K}$ over the four epochs. Both this strong rise and the fact that the earlier estimates are below the lower inverse Compton limit can be interpreted as indicators of relativistic beaming.

Furthermore we calculated the equipartition Doppler factor $\delta_{\text {equ }}$ following Readhead (1994) and Güijosa \& Daly (1996), which implies that the radiating particles have the same energy as the penetrating magnetic field. The results (assuming as above $h=0.71$ )

$\delta_{\text {equ }}=\left(0.5_{-0.3}^{+2.6}\right)$

\footnotetext{
5 If much more (spectral and multi-epoch) data are available as e.g. in the case of the quasar 3C 345, inhomogeneities in the particle number density can also be estimated. Lobanov \& Zensus (1999) used more detailed analyzis to explain the observed flux density variations quantitatively with time of $3 \mathrm{C} 345$.
}

are very similar to the inverse-Compton Doppler factor (Table 8). This supports Readhead's conclusion that many powerful, non-thermal extragalactic radio sources are close to energy equipartition and their finding that the brightness temperatures of the respective sources range far below the maximum brightness temperature of $10^{12} \mathrm{~K}$ (see above).

As in the previous section, we now adopt that the intrinsic velocities along the jet are equal to the highest minimal intrinsic velocity, as given by the apparent separation speeds. Then the angle between the direction of motion and the line of sight can be estimated from the Doppler factor:

$\delta_{\text {core }}=\left(0.5_{-0.3}^{+1.8}\right) ; \beta_{\text {min }}^{\mathrm{A}} \approx\left(0.999_{-0.0005}^{+0.0003}\right) \rightarrow \vartheta_{\text {core }} \approx\left(24_{-14}^{+19}\right)^{\circ}$.

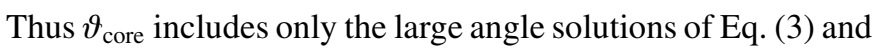
supports the idea of a straight inner jet nozzle as described in Stirling et al. (2003).

\section{Discussion of the results}

For the first time the kinematics of the non-thermal radio jet components of $\mathrm{J} 1101+7225$ were estimated. We calculated the apparent motions of the two extended components, observable at low GHz-frequencies from VLBI-measurements. We found an apparent superluminal separation speed of $\beta_{\text {app }}($ Core $-A)=$ $(22.5 \pm 4)$ for the A-component over the past decade at an exceptionally large deprojected distance to the core (22 mas $4 \mathrm{kpc}$ ). The even more distant B-component was found to be stationary within the errors at a core-distance of $\sim 53$ mas.

Furthermore a Doppler factor $\delta=\left(0.5_{-0.3}^{+1.8}\right)$ was estimated for the compact optically thick (at the observing frequencies) synchrotron radiation of the core of $\mathrm{J} 1101+7225$. The strength of the magnetic field, which is necessary for the synchrotron process, was estimated to be $B_{0}=\left(0.15_{-0.12}^{+0.2}\right) \mathrm{G}$. The estimated size of the optically thick nuclear component is close to the resolution limit of an interferometric observation at cmwavelengths, which suggests, in combination with the estimated Doppler factor and intrinsic jet velocities, that additional VLBI-components could appear outside the core in the future.

More observations of these nuclear components could reveal, if a deceleration of the A-component appears while A is approaching the B-component. This would be similar to the well known case of 4C 39.25 (Alberdi et al. 1993a). Deceleration at these distances from the core can confirm an interaction of the jet component with the circumnuclear matter of the host galaxy as described by Taylor et al. (1989) for Seyfert nuclei. Such an interaction is strongly supported by comparing the VLBI maps presented here with the jet geometry as observed with the VLA (Xu et al. 1995). In their 1.4 GHz map two corresponding radio jets appear, extending along $\sim 5^{\prime \prime}$ from the core. While the southwestern jet extends in the opposite direction of the VLBI jet, the northeastern VLA-jet does not coincide with the position angle of the VLBI structure. Instead it appears to be bent towards a northwesternly direction, perhaps induced by ram pressure of the surrounding material.

Apparent superluminal velocities are explained by motion towards the observer at relativistic velocity. The additionally presented $5 \mathrm{GHz}$ maps and Gaussian modelfits of the central 5 mas show further jet components with significantly 
smaller apparent velocities. Thus the separation velocities of the different VLBI radio jet components of J1101+7225 show no simple correlation with their distance to the core. But this does not rule out a constant intrinsic velocity, because already small variations of the angle between jet and the line of sight can introduce variations of the apparent speeds of the observed order of magnitude. In the case of such a constant intrinsic velocity along the whole jet, the very high velocity, derived from the A-component, has to be chosen. Such high intrinsic velocities explain both the large extension of the radio jet over several arcsec as estimated with the VLA and the high luminosity of the core.

We believe that our findings may indicate a helical bending of the jet, where the fast components are moving in a section that is curved towards the observer. This is supported by the estimated differing jet orientations with respect to the line of sight. Zensus et al. (1995) could explain the acceleration of a jet component of 3C 345 by a curved jet of constant intrinsic bulk velocity. It has been shown that helical jet patterns result from Kelvin-Helmholtz and current-driven jet instabilities in relativistic flows (Birkinshaw 1991; Istomin \& Pariev 1996).

Beside this interpretation of the observed motion as an intrinsic bulk motion of the radiating plasma, shock waves may travel along the jet (Alberdi et al. 1993b). Different component velocities may also be observed if the slower components are trailing in the wake of faster ones. This hydrodynamical explanation could be adopted successfully to the complex component motion in the radio jet of the radio galaxy 3C 120 (Gomez et al. 2001). Hardee et al. (2001) found mechanisms to produce differentially moving and stationary features in a jet by analyzing the relativistic hydrodynamic equations.

Thus the nuclear region of $\mathrm{J} 1101+7225$ provides the rare possibility of observing the total range of jet kinematics including apparent superluminal separation velocities even far out of the central parsec-region. The results presented here can give observational constraints far from the jet origin for numerical jet models.

Acknowledgements. We are grateful to the correlator team of the MPIfR in Bonn for their assistance. The European VLBI Network is a joint facility of European, Chinese, South African, and other radio astronomy institutes funded by their national research councils. The National Radio Astronomy Observatory is a facility of the National Science Foundation operated under cooperative agreement by Associated Universities, Inc. This work was supported in part by the Deutsche Forschungsgemeinschaft (DFG) via grant SFB 494.

\section{References}

Alberdi, A., Krichbaum, T. P., Marcaide, J. M., et al. 1993a, A\&A, 271, 93

Alberdi, A., Marcaide, J. M., Marscher, A. P., et al. 1993b, ApJ, 402, 160

Alef, W., \& Graham, D. A. 2002, in New Developments in VLBI Science and Technology, ed. E. Ros, R. W. Porcas, A. P. Lobanov, \& J. A. Zensus (Max-Planck-Institut für Radioastronomie, Bonn), 31

Beasley, A. J., Gordon, D., Peck, A. B., et al. 2002, ApJS, 141, 13 (B02)
Birkinshaw, M. 1991, in Beams and Jets in Astrophysics, ed. P. A. Hughes (Cambridge University Press), 278

Blandford, R. D., \& Königl, A. 1979, ApJ, 232, 34

Bloom, S. D., \& Marscher, A. P. 1991, ApJ, 366, 16

Brinkmann, W., Yuan, W., \& Siebert, J. 1997, A\&A, 319, 413

Britzen, S. 2002, Rev. Mod. Astron., 15, 199

Fiore, F., Giommi, P., Vignali, C., et al. 2001, MNRAS, 327, 771

Fomalont, E. B. 1989, in Synthesis imaging in radio astronomy, ed. R. A. Perley, F. R. Schwab, \& A. H. Bridle (ASP), 6, 213

Gómez, J., \& Marscher, A. P. 2000, ApJ, 530, 245

Gómez, J., Marscher, A. P., Alberdi, A., Jorstad, S. G., \& Agudo, I. 2001, ApJ, 561, L161

Gower, J. F. R., Scott, P. F., \& Wills, D. 1967, MmRAS, 71, 49

Gregory, P. C., \& Condon, J. J. 1991, ApJS, 75, 1011

Gregory, P. C., Scott, W. K., Douglas, K., \& Condon, J. J. 1996, ApJS, 103,427

Güijosa, A., \& Daly, R. A. 1996, ApJ, 461, 600

Hales, S. E. G., Waldram, E. M., Rees, N., \& Warner, P. J. 1995, MNRAS, 274, 447

Hardee, P. E., Hughes, P. A., Rosen, A., \& Gomez, E. A. 2001, ApJ, 555,744

Hummel, C. A., Muxlow, T. W. B., Krichbaum, T. P., et al. 1992, A\&A, 266, 93

Istomin, Y. N., \& Pariev, V. I. 1996, MNRAS, 281, 1

Jackson, N., \& Browne, I. W. A. 1991, MNRAS, 250, 414

Königl, A. 1981, ApJ, 243, 700

Kraus, J. D. 1986, Radio Astronomy (Powell, Ohio: Cygnus-Quasar Books), Chap. 10

Kühr, H., Pauliny-Toth, I. I. K., Witzel, A., \& Schmidt, J. 1981, AJ, 86,854

Lobanov, A. P., \& Zensus, J. A. 1999, ApJ, 521, 509

Marscher, A. P. 1983, ApJ, 264, 296

Marscher, A. P. 1988, ApJ, 334, 552

Otterbein, K., Krichbaum, T. P., Kraus, A., et al. 1998, A\&A, 334, 489

Patnaik, A. R., Browne, I. W. A., Wilkinson, P. N., \& Wrobel, J. M. 1992, MNRAS, 254, 655

Pauliny-Toth, I. I. K. 1998, in Radio Emission from Galactic and Extragalactic Compact Sources, ed. J. A. Zensus, G. B. Taylor, \& J. M. Wrobel, ASP Conf. Ser., 144, 75

Pearson, T. J., \& Zensus, J. A. 1987, in Superluminal Radio Sources, ed. J. A. Zensus, \& T. J. Pearson (Cambridge University Press), 1

Pearson, T. J., Shepherd, M. C., Taylor, G. B., \& Myers, S. T. 1994, BAAS, 26, 1318

Pearson, T. J. 1995, in Very Long Baseline Interferometry and the VLBA, ed. J. A. Zensus, P. J. Diamond, \& P. J. Napier (San Francisco: ASP), 268

Readhead, A. C. S. 1994, ApJ, 426, 51

Rees, N. 1990, MNRAS, 244, 233

Reich, W., Fürst, E., Reich, P., et al. 2000, A\&A, 363, 141

Stirling, A. M., Cawthorne, T. V., Stevens, J. A., et al. 2003, MNRAS, 341,405

Taylor, D., Dyson, J. E., Axon, D. J., \& Pedlar, A. 1989, MNRAS, 240, 487

Taylor, G. B., Vermeulen, R. C., Readhead, A. C. S., et al. 1996, ApJS, 107,37

Teräsranta, H., Urpo, S., Wiren, S., \& Valtonen, M. 2001, A\&A, 368, 431

Thakkar, D. D., Xu, W., Readhead, A. C. S., et al. 1995, ApJS, 98, 33 (T95)

Véron-Cetty, M.-P., \& Véron, P. 2001, A\&A, 374, 92

White, R. L., \& Becker, R. H. 1992, ApJS, 79, 331

Xu, W., Readhead, A. C. S., Pearson, T. J., Polatidis, A. G., \& Wilkinson, P. N. 1995, ApJS, 99, 297

Zensus, J. A., Cohen, M. H., \& Unwin, S. C. 1995, ApJ, 443, 35 